\title{
Data-Driven Networked Optimal Iterative Learning Control for Discrete Linear Time-Varying Systems with One-Operation Bernoulli-Type Communication Delays
}

\author{
Yan Geng, ${ }^{1}$ Xiaoe Ruan, ${ }^{1}$ and Hyo-Sung $\mathrm{Ahn}^{2}$ \\ ${ }^{1}$ Department of Applied Mathematics, School of Mathematics and Statistics, Xian Jiaotong University, Xian, China \\ ${ }^{2}$ School of Mechanical Engineering, Gwangju Institute of Science and Technology (GIST), Gwangju 500-712, Republic of Korea \\ Correspondence should be addressed to Xiaoe Ruan; wruanxe@mail.xjtu.edu.cn
}

Received 8 September 2016; Accepted 22 January 2017; Published 6 March 2017

Academic Editor: Guang Zhang

Copyright (C) 2017 Yan Geng et al. This is an open access article distributed under the Creative Commons Attribution License, which permits unrestricted use, distribution, and reproduction in any medium, provided the original work is properly cited.

\begin{abstract}
This paper develops a type of data-driven networked optimal iterative learning control strategy for a class of discrete linear timevarying systems with one-operation Bernoulli-type communication delays. In terms of the stochastic Bernoulli-type one-operation communication delayed inputs and outputs, the previous-iteration synchronous compensations are adopted. By means of deriving gradients of two types of objective functions that express the optimal approximation of the system matrix and the minimal tracking error, the strategy approximates the system matrix and upgrades the control inputs in an interact mode as the iteration evolves. By taking advantage of matrix theory and statistical technique, it is derived that the approximation discrepancy of the system matrix is bounded and the mathematical expectation of the tracking error vanishes as the iteration goes on. Numerical simulations manifest the validity and effectiveness.
\end{abstract}

\section{Introduction}

Iterative learning control (ILC) has been acknowledged as one of effectively intelligent strategies, which performs a high-precision trajectory tracking repetitively over a fixed time interval, as surveyed in [1-3]. Since its invention, numerous ILC strategies have been developed for theoretical analyses and practical applications over the past three decades [4-11]. Convergence analyses as one of key ILC theoretical issues have been discussed from all aspects of the norm of tracking errors for guaranteeing the ILC implement, such as infinite-norm [4], lambda-norm [5], sup-norm [6], Lebesgue- $p$ norm [7], 2D technique [8], and Lyapunov method [9]. Form practical executions, kinds of system perturbations and uncertainties are avoidable, such as iterationvarying disturbances, time-varying uncertainties, and system uncertainty. For that, robust ILCs have been involved in [1013]. It is reminded that, basically, the strategic feature of the ILC is that its formulation is irrelevant to the system dynamics but the multioperation inputs and outputs. However, in industry applications, model-based ILC can perform better than without any system information ILC, where at least an approximate model is needed. This implies that the ILC may be regarded as a data-driven scheme which utilizes the historical inputs, outputs, and model-approximation to formulate a sequence of updating control inputs. Thus, the terminology of the data-driven ILC has been emerged, such as [14-16].

A data-driven terminal ILC approach has been proposed for a kind of linear discrete-time-varying system in [14], where the convergence is derived under the assumption that the product of system matrix and approximated matrix is positively definite. In addition, [15] has presented a data-driven constrained norm-optimal ILC and then approximated the impulse response of the system by measurements of inputs and outputs for linear time-invariant systems. Further, [16] has presented a data-driven predictive ILC scheme based on a dynamic linearization technique for a class of discretetime nonlinear systems, whose convergence is ensured under the requirement that the approximated matrix is diagonally 
dominant. The data-driven ILCs mentioned-above work well. However, the requirements that approximation matrix is positively definite or diagonally dominant, however, are quite rigorous. This may confine the feasibility of the schemes.

On the other hand, with the advancement of internet technology, networked control systems (NCSs) have been mushroomed ranging from industrial manufacturing to modern medical technology and so on owing to its lower cost, simple installation, easy maintenance, high reliability, and convenient source sharing [17]. However, in NCSs, the communication delay or data dropout is inevitable due to communication constraints, network congestions, or other factors. These may influence the stability and performance of the controlled system. In the field of NCSs, the major focus is to compensate the communication delayed or dropped data by some appropriate techniques so as to maintain the performance of systems [18]. In particular, for the case when the ILC scheme is implemented through the network to compensate the data, the mode is regarded as a networked ILC scheme, such as [19-22]. For the facet, [19] has first introduced a networked ILC scheme to deal with the random data delays and dropouts, where the convergence and stability were analyzed in the mean-square sense for discrete linear time-invariant systems. Thereafter, [20] has proposed a networked ILC approach to a class of nonlinear systems for the network-communicated input and output signals with constant time delays and stochastic packet loss. Recently, in [21], a compensated ILC has been provided for a class of nonlinear systems with random one-step communication delays. Further, two types of compensation schemes are employed for linear discrete-time stochastic systems with one-step communication delays in [22].

However, the existing networked ILC schemes for handing communication delays are almost all required accurate system model within one-step time internal. The requirements of the system dynamics and one-step time delay are rigorous. It is worth minding that the data-driven ILC utilizes the multi-iteration inputs and outputs to construct the updating law in a recursive mode. This implies that the so-called open-loop ILC makes it possible to relax the communication delay within one-operation period. These motivate the paper to develop a data-driven optimal ILC scheme for a class of discrete linear time-varying systems with one-operation communication delays. Differing from the data-driven ILCs in [14-16], this paper compensates for the delayed Bernoulli-type inputs and outputs by its previousiteration synchronous data and analyzes the convergence of the approximation benefiting from matrix theory with no requirement of positively definite or diagonal dominance.

The paper is organized as follows. Section 2 firstly gives the description of networked control systems and provides compensations for one-operation communication delayed data in the form of super vectors and then develops the data-driven networked optimal ILC with the compensation strategy. In Section 3, the convergences of approximation discrepancy and tracking error are derived, respectively. Numerical simulations are illustrated in Section 4 to exhibit the validity and the effectiveness and the last Section 5 concludes the paper.

\section{Data-Driven Networked Optimal ILC Scheme}

2.1. Networked Control Systems and Data Compensations. Throughout the paper, the 2-norm for a vector $x \in R^{N}$ is defined as $\|x\|_{2}=\sqrt{x^{T} x}$ and the induced 2-norm for a matrix $\mathbf{M} \in R^{N \times N}$ is expressed as $\|\mathbf{M}\|_{2}=\sqrt{\max _{i=1,2, \ldots, N}\left(\lambda_{i}\left(\mathbf{M}^{T} \mathbf{M}\right)\right)}$, where the superscript " $T$ " refers to the transpose operation and $\lambda_{i}\left(\mathbf{M}^{T} \mathbf{M}\right), i=1,2, \ldots, N$, are eigenvalues of the matrix $\mathbf{M}^{T} \mathbf{M}$.

Consider a class of repetitive discrete linear time-varying SISO systems described as follows:

$$
\begin{aligned}
x_{k}(t+1) & =\mathbf{A}(t) x_{k}(t)+\mathbf{B}(t) u_{k}(t), \\
y_{k}(t) & =\mathbf{C}(t) x_{k}(t)
\end{aligned}
$$

where $t \in\{0,1, \ldots, N\}$ represents the sampling instant, $N$ is the total of instant numbers, and $k=1,2, \ldots$ is the operation index. $x_{k}(t) \in R^{n}, u_{k}(t) \in R$, and $y_{k}(t) \in R$ are $n$-dimensional state vector, scalar input, and scalar output at the $k$ th operation, respectively. $\mathbf{A}(t), \mathbf{B}(t)$, and $\mathbf{C}(t)$ are unknown time-varying but bounded matrices with appropriate dimensions.

Given that $y_{d}(t), t \in\{0,1, \ldots, N\}$, is a predetermined desired trajectory, the data-driven networked optimal ILC scheme is constructed under the assumptions listed as follows.

(A1) The desired trajectory $y_{d}(t), t \in\{0,1, \ldots, N\}$, is realizable; that is, there exist such desired state $x_{d}(t)$ and desired input $u_{d}(t)$ that

$$
\begin{aligned}
x_{d}(t+1) & =\mathbf{A}(t) x_{d}(t)+\mathbf{B}(t) u_{d}(t) \\
y_{d}(t) & =\mathbf{C}(t) x_{d}(t)
\end{aligned}
$$

(A2) For repetitive system (1), the initial state is resettable. Namely, the resetting condition $x_{k}(0)=x_{d}(0)$ holds for all operation indices $k=1,2, \ldots$, where $x_{d}(0)$ is the initial desired state. Without loss of generality, we set $x_{d}(0)=0$.

(A3) The stochastic communication data delays are subject to Bernoulli-type distributions but confined within one-operation period.

It is known that in a closed-loop networked control system, the output signal is transmitted from the sensor to the controller through output communication channel, and simultaneously, the input signal is delivered from the controller to the actuator through input communication channel, respectively. Likewise, the data-driven networked optimal iterative learning control (DDNOILC) scheme for system (1) works in the same mode. Its schematic paradigm is exhibited in Figure 1.

In Figure 1, denote $\tilde{u}_{k}(t)$ as the current-iteration $t$ thinstant control input generated from the ILC controller which is transmitted to the actuator via input communication channel, whilst let $u_{k}(t)$ be the current-iteration $t$ th-instant 


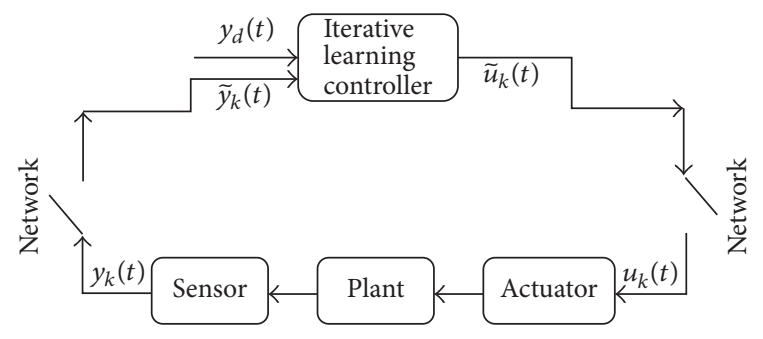

FIgURE 1: The schematic diagram of DDNOILC.

control input of the actuator for plant stimulation, which is either equal to $\widetilde{u}_{k}(t)$ in the case when the data $\tilde{u}_{k}(t)$ is timely transmitted in success with the probability $a$ or equal to the previous-iteration synchronous $\widetilde{u}_{k-1}(t)$ in the case when the transmission of data $\widetilde{u}_{k}(t)$ is delayed within oneoperation period with the probability $1-a$. Mathematically, a compensation for $u_{k}(t)$ is expressed as

$$
u_{k}(t)=\alpha_{k}(t) \tilde{u}_{k}(t)+\left(1-\alpha_{k}(t)\right) \tilde{u}_{k-1}(t),
$$

where $\alpha_{k}(t)$ is independent stochastic variable subject to $0-1$ Bernoulli-type distribution with

$$
\begin{aligned}
& P\left\{\alpha_{k}(t)=1\right\}=E\left\{\alpha_{k}(t)\right\}=a, \\
& P\left\{\alpha_{k}(t)=0\right\}=1-E\left\{\alpha_{k}(t)\right\}=1-a,
\end{aligned}
$$

where $P\{\cdot\}$ and $E\{\cdot\}$ are probability and expectation of $\{\cdot\}$, respectively. Parameter $a$ is a priori probability satisfying $0<$ $a \leq 1$. The equation $\alpha_{k}(t)=1$ means that the signal $\tilde{u}_{k}(t)$ is transmitted timely to the plant, whilst the equation $\alpha_{k}(t)=$ 0 implies that the signal $\widetilde{u}_{k}(t)$ is delayed and the previousiteration signal $\tilde{u}_{k-1}(t)$ is used to compensate for the delayed data.

Simultaneously, the employed data $\tilde{y}_{k}(t)$ for the ILC updating is equal to either $y_{k}(t)$ or $y_{k-1}(t)$ in the Bernoullitype switch. Mathematically, a compensation for $\tilde{y}_{k}(t)$ is expressed as

$$
\tilde{y}_{k}(t)=\beta_{k}(t) y_{k}(t)+\left(1-\beta_{k}(t)\right) y_{k-1}(t),
$$

where $\beta_{k}(t)$ is independent stochastic variable subject to $0-1$ Bernoulli-type distribution with

$$
\begin{aligned}
& P\left\{\beta_{k}(t)=1\right\}=E\left\{\beta_{k}(t)\right\}=b, \\
& P\left\{\beta_{k}(t)=0\right\}=1-P\left\{\beta_{k}(t)=1\right\}=1-b,
\end{aligned}
$$

where parameter $b$ is a priori probability satisfying $0<b \leq 1$.

The implication of $\beta_{k}(t)$ is similar with $\alpha_{k}(t)$. Moreover, the variables $\alpha_{k}(t)$ and $\beta_{k}(t)$ are independent of each other; that is,

$$
\begin{aligned}
& P\left\{\alpha_{k}(t) \beta_{k}(t)\right\}=P\left\{\alpha_{k}(t)\right\} P\left\{\beta_{k}(t)\right\}, \\
& E\left\{\alpha_{k}(t) \beta_{k}(t)\right\}=E\left\{\alpha_{k}(t)\right\} E\left\{\beta_{k}(t)\right\} .
\end{aligned}
$$

Denote

$$
\begin{aligned}
& u_{k}=\left[u_{k}(0), u_{k}(1), \ldots, u_{k}(N-1)\right]^{T}, \\
& y_{k}=\left[y_{k}(1), y_{k}(2), \ldots, y_{k}(N)\right]^{T}, \\
& u_{d}=\left[u_{d}(0), u_{d}(1), \ldots, u_{d}(N-1)\right]^{T}, \\
& y_{d}=\left[y_{d}(1), y_{d}(2), \ldots, y_{d}(N)\right]^{T}, \\
& \tilde{u}_{k}=\left[\tilde{u}_{k}(0), \tilde{u}_{k}(1), \ldots, \widetilde{u}_{k}(N-1)\right]^{T}, \\
& \tilde{y}_{k}=\left[\tilde{y}_{k}(1), \tilde{y}_{k}(2), \ldots, \tilde{y}_{k}(N)\right]^{T} .
\end{aligned}
$$

Then, system (1) is reformulated in a super vector form as

$$
y_{k}=\mathbf{H} u_{k}
$$

where $\mathbf{H}=\left(h^{i j}\right)_{N \times N}$ is a lower triangular matrix specified as follows:

$$
\begin{array}{r}
h^{i j}= \begin{cases}0, & i<j, \\
\mathbf{C}(i) \prod_{m=j}^{i-1} \mathbf{A}(m) \mathbf{B}(j-1), & i \geq j,\end{cases} \\
\prod_{m=j}^{i-1} \mathbf{A}(m)= \begin{cases}\mathbf{I}, & i=j, \\
\mathbf{A}(i-1) \mathbf{A}(i-2) \cdots \mathbf{A}(j), & i>j .\end{cases}
\end{array}
$$

From (A1) and (A2), it is easy to obtain

$$
y_{d}=\mathbf{H} u_{d}
$$

Further, expressions (3) and (5) can be rewritten as

$$
\begin{aligned}
& u_{k}=\Psi_{k} \tilde{u}_{k}+\bar{\Psi}_{k} \tilde{u}_{k-1}, \\
& \tilde{y}_{k}=\Phi_{k} y_{k}+\overline{\boldsymbol{\Phi}}_{k} y_{k-1},
\end{aligned}
$$

where

$$
\begin{aligned}
& \boldsymbol{\Psi}_{k}=\operatorname{diag}\left(\alpha_{k}(1) \alpha_{k}(2) \cdots \alpha_{k}(N)\right), \\
& \boldsymbol{\Phi}_{k}=\operatorname{diag}\left(\beta_{k}(1) \beta_{k}(2) \cdots \beta_{k}(N)\right), \\
& \bar{\Psi}_{k}=\mathbf{I}-\Psi_{k}, \\
& \overline{\boldsymbol{\Phi}}_{k}=\mathbf{I}-\boldsymbol{\Phi}_{k},
\end{aligned}
$$

and $\mathbf{I}$ is $N \times N$ identity matrix.

Note that the lower triangular matrix $\mathbf{H}$ is unknown but bounded due to the fact that matrices $\mathbf{A}(t), \mathbf{B}(t)$, and $\mathbf{C}(t)$ are unknown but bounded.

2.2. Data-Driven Networked Optimal ILC. In terms of constructing an optimal ILC updating law of the control command $u_{k+1}$ in a recursive form for system (9), the ordinary way is to raise an objective function and to solve it by an approximate manner. This implies that the system matrix $\mathbf{H}$ must be available. But, in usual, the system matrix $\mathbf{H}$ is 
hardly to be available due to the complexity of the system modeling. Nevertheless, since the fundamental ILC scheme is to make use of the tracking error to upgrade its control input as the operation repeats, it is thus possible that, in composing the optimal ILC updating rule, the system matrix $\mathbf{H}$ may be substituted by an appropriated one. Meanwhile, the approximation of the system matrix may be updated benefiting from the upgraded control input as well. This composes an interacted updating fashion of the system matrix and the control inputs detailed in the following.

Let $\mathbf{H}_{k}$ be the $k$ th approximation of $\mathbf{H}$.

Denote

$$
\begin{aligned}
\mathbf{H} & =\left[\left(H^{1}\right)^{T}\left|\left(H^{2}\right)^{T}\right| \cdots \mid\left(H^{N}\right)^{T}\right]^{T}, \\
\mathbf{H}_{k} & =\left[\left(H_{k}^{1}\right)^{T}\left|\left(H_{k}^{2}\right)^{T}\right| \cdots \mid\left(H_{k}^{N}\right)^{T}\right]^{T},
\end{aligned}
$$

where $\left(H^{i}\right)^{T}$ and $\left(H_{k}^{i}\right)^{T}$ are the $i$ th row of matrices $\mathbf{H}$ and $\mathbf{H}_{k}$, respectively.

It is evident that $\left(H_{k}^{i}\right)^{T}$ is the $k$ th approximation of $\left(H^{i}\right)^{T}$ for all $i=1, \ldots, N$. There is no doubt that the postulation $\lim _{k \rightarrow \infty}\left(H_{k}^{i}\right)^{T}=\left(H^{i}\right)^{T}$, for all $i=1, \ldots, N$, is equivalent to that of $\lim _{k \rightarrow \infty} \mathbf{H}_{k}=\mathbf{H}$. Besides, description (9) is no other but $y_{k}(i)=\left(H^{i}\right)^{T} u_{k}$.

For the purpose of generating an updating law to update the approximated vector $\left(H_{k}^{i}\right)^{T}$ in an optimal sense, consider an optimization problem as

$$
\begin{aligned}
\min _{\left(H_{k-1}^{i}\right)^{T}} J & \left(\left(H_{k-1}^{i}\right)^{T}\right)=\frac{1}{2}\left|y_{k}(i)-\left(H_{k-1}^{i}\right)^{T} u_{k}\right|^{2} \\
= & \frac{1}{2}\left(H_{k-1}^{i}\right)^{T} u_{k} u_{k}^{T} H_{k-1}^{i}-y_{k}(i) u_{k}^{T} H_{k-1}^{i} \\
& +\frac{1}{2}\left(y_{k}(i)\right)^{2} .
\end{aligned}
$$

The gradient of objective function (15) with respect to $\left(H_{k-1}^{i}\right)^{T}$ is expressed as

$$
\nabla J\left(\left(H_{k-1}^{i}\right)^{T}\right)=-\left(y_{k}(i)-\left(H_{k-1}^{i}\right)^{T} u_{k}\right) u_{k}^{T} .
$$

Thus, the gradient-type updating formula for $\left(H_{k}^{i}\right)^{T}$ is given by

$$
\left(H_{k}^{i}\right)^{T}=\left(H_{k-1}^{i}\right)^{T}+\gamma_{k}\left(y_{k}(i)-\left(H_{k-1}^{i}\right)^{T} u_{k}\right) u_{k}^{T},
$$

where $\gamma_{k}$ is the updating step. Substituting (17) into (15), it has

$$
\begin{array}{rl}
\min _{\gamma_{k}} & J\left(\gamma_{k}\right) \\
& =\frac{1}{2}\left[\left(y_{k}(i)-\left(H_{k-1}^{i}\right)^{T} u_{k}\right)\left(1-\gamma_{k} u_{k}^{T} u_{k}\right)\right]^{2} .
\end{array}
$$

Obviously, the optimal step is $\gamma_{k}=1 / u_{k}^{T} u_{k}=1 /\left\|u_{k}\right\|_{2}^{2}$. For ensuring the convergence and strengthening the practicability of the gradient-type algorithm (17), select $\gamma_{k}$ as

$$
\gamma_{k}=\frac{1}{\mu+\left\|u_{k}\right\|_{2}^{2}},
$$

where $\mu$ is a relaxing factor satisfying $\mu>0$, which is adopted for guaranteeing the denominator of $\gamma_{k}$ being nonzero as $u_{k}=$ 0 .

Equivalently, by (17), $\mathbf{H}_{k}$ is updated by the following:

$$
\mathbf{H}_{k}=\mathbf{H}_{k-1}+\gamma_{k}\left(y_{k}-\mathbf{H}_{k-1} u_{k}\right) u_{k}^{T} .
$$

It is hopeful that updating law (20) may improve the approximation as the iteration number increases. The approximation discrepancy $\Delta \mathbf{H}_{k}$ is defined as $\Delta \mathbf{H}_{k}=\mathbf{H}-\mathbf{H}_{k}$.

Mind that the purpose of the data-driven optimal ILC scheme is to construct an updating law of the control command $u_{k+1}$ in a recursive form in order to optimize an objective function. By taking the above-mentioned approximation into account, consider an optimization problem as follows:

$$
\begin{array}{rl}
\min _{u_{k}} & J\left(u_{k}\right)=\frac{1}{2}\left\|e_{k}\right\|_{2}^{2}=\frac{1}{2}\left\|y_{d}-\mathbf{H}_{k} u_{k}\right\|_{2}^{2} \\
= & \frac{1}{2} u_{k}^{T} \mathbf{H}_{k}^{T} \mathbf{H}_{k} u_{k}-y_{d}^{T} \mathbf{H}_{k} u_{k}+\frac{1}{2} y_{d}^{T} y_{d},
\end{array}
$$

where $e_{k}$ is the tracking error defined as $e_{k}=y_{d}-y_{k}$, meanwhile $\mathbf{H}$ in $y_{k}=\mathbf{H} u_{k}$ is substituted by $\mathbf{H}_{k}$.

The gradient of (21) with respect to $u_{k}$ is derived as

$$
\nabla J\left(u_{k}\right)=-\mathbf{H}_{k}^{T} e_{k} .
$$

Thus, the gradient-type algorithm for the control input is derived as

$$
u_{k+1}=u_{k}+\pi_{k} \mathbf{H}_{k}^{T} e_{k},
$$

where $\pi_{k}$ is learning gain. Substituting (23) into (21), it obtains

$$
\min _{\pi_{k}} J\left(\pi_{k}\right)=\frac{1}{2}\left\|\left(\mathbf{I}-\pi_{k} \mathbf{H}_{k} \mathbf{H}_{k}^{T}\right) e_{k}\right\|_{2}^{2} .
$$

As the matrix $\mathbf{H}_{k} \mathbf{H}_{k}^{T}$ is symmetric and nonnegative definite, all its eigenvalues are nonnegative. Thus, it is easy to induce

$$
\begin{aligned}
\| \mathbf{I} & -\pi_{k} \mathbf{H}_{k} \mathbf{H}_{k}^{T} \|_{2}^{2}=\max _{1 \leq i \leq N}\left\{\left|\lambda_{i}\left(\mathbf{I}-\pi_{k} \mathbf{H}_{k} \mathbf{H}_{k}^{T}\right)\right|\right\} \\
& =\max _{1 \leq i \leq N}\left\{\left|1-\pi_{k} \lambda_{i}\left(\mathbf{H}_{k} \mathbf{H}_{k}^{T}\right)\right|\right\} \\
& =\max \left\{\left|1-\pi_{k} \lambda_{\min }\left(\mathbf{H}_{k} \mathbf{H}_{k}^{T}\right)\right|,\right. \\
& \left.\left|1-\pi_{k} \lambda_{\max }\left(\mathbf{H}_{k} \mathbf{H}_{k}^{T}\right)\right|\right\},
\end{aligned}
$$

where $\lambda_{i}(\cdot)$ is the $i$ th eigenvalues of $(\cdot)$ and $\lambda_{\max }(\cdot)$ and $\lambda_{\text {min }}(\cdot)$ are the maximal and minimal eigenvalues of $(\cdot)$, respectively.

Obviously, for ensuring the convergence of the gradienttype algorithm (23), $\pi_{k}$ is selected as

$$
\pi_{k}=\frac{1}{\varepsilon+\lambda_{\max }\left(\mathbf{H}_{k} \mathbf{H}_{k}^{T}\right)},
$$

where $\varepsilon>0$ is a weighing factor. 
Combining the above-mentioned updating laws (20) and (23) together, a data-driven optimal ILC (DDOILC) is constructed for system (9) as follows:

$$
\begin{aligned}
\mathbf{H}_{k} & =\mathbf{H}_{k-1}+\gamma_{k}\left(y_{k}-\mathbf{H}_{k-1} u_{k}\right) u_{k}^{T}, \\
u_{k+1} & =u_{k}+\pi_{k} \mathbf{H}_{k}^{T} e_{k} .
\end{aligned}
$$

Remark 1. The control input updating law (28) is derived for the special case in Figure 1 when the communication delays do not occur as formulated as $a=b=1$. This implies that the plant inputs are equal to the ILC updated inputs and, meanwhile, the plant outputs are equal to the employed outputs; that is, $u_{k}=\widetilde{u}_{k}$ and $\widetilde{y}_{k}=y_{k}$, respectively.

Remark 2. It should be pointed that, for system (1), the system matrices $\mathbf{A}(t), \mathbf{B}(t)$, and $\mathbf{C}(t)$ are assumed to be time-varying. For the circumstance, the parameter $\mathbf{H}$ is lower triangular matrix. As such, for an ideal approximation, it is desirable to guarantee the matrix $\mathbf{H}_{k}, k \geq 2$, to be lower triangular. But this is not easily realizable. However, updating law (27) can insure that the discrepancy of the approximation $\mathbf{H}_{k}$ from the system matrix $\mathbf{H}$ is monotonously convergent to a bounded constant.

By considering (A3) for the cases $0<a<1$ and $0<$ $b<1$ when the stochastic communication data delays are subject to Bernoulli-type distributions but confined within one-operation period, the ILC controller for system (9) with one-operation communication delays is designed as

$$
\widetilde{u}_{k+1}=\widetilde{u}_{k}+\pi_{k} \mathbf{H}_{k}^{T} \widetilde{e}_{k},
$$

where $\widetilde{e}_{k}$ is the tracking error defined as $\widetilde{e}_{k}=y_{d}-\widetilde{y}_{k}$.

Consequently, a data-driven networked optimal ILC (DDNOILC) algorithm is developed for system (9) with oneoperation communication delays as follows:

$$
\begin{aligned}
\widetilde{u}_{1}=u_{1} \text { and } \widetilde{y}_{1}=y_{1} \text { : given test signals; } \\
\mathbf{H}_{1} \text { : arbitrarily given and nonsingular; } \\
\mu>0 \text { and } \varepsilon>0 \text { : set appropriately. } \\
u_{k}=\boldsymbol{\Psi}_{k} \widetilde{u}_{k}+\bar{\Psi}_{k} \widetilde{u}_{k-1}, \\
\gamma_{k}=\frac{1}{\mu+\left\|u_{k}\right\|_{2}^{2}}, \\
\mathbf{H}_{k}=\mathbf{H}_{k-1}+\gamma_{k}\left(y_{k}-\mathbf{H}_{k-1} u_{k}\right) u_{k}^{T}, \\
\widetilde{y}_{k}=\boldsymbol{\Phi}_{k} y_{k}+\overline{\boldsymbol{\Phi}}_{k} y_{k-1}, \\
\widetilde{e}_{k}=y_{d}-\widetilde{y}_{k}, \\
\pi_{k}=\frac{1}{\varepsilon+\lambda_{\max }\left(\mathbf{H}_{k} \mathbf{H}_{k}^{T}\right)}, \\
\widetilde{u}_{k+1}=\widetilde{u}_{k}+\pi_{k} \mathbf{H}_{k}^{T} \widetilde{e}_{k} .
\end{aligned}
$$

Notice that the DDNOILC scheme (30)-(36) is only related to the data $u_{k}, y_{k}, \tilde{y}_{k}$, and $\tilde{u}_{k}$.
Remark 3. It is noticed that the derivations of updating laws (27) and (28) are from the gradient-type mechanism, which are prominently distinct from the existing datadriven ILCs in [14-16] which searches Kuhn-Tucker point. The method avoids the complex computation of matrix inversion. Significantly, the derivations of the paper do not rigorously require that the approximation matrices must be diagonally dominant or the product of system matrix and approximated matrix is positively definite. Moreover, the developed DDNOILC scheme (30)-(36) may deal with the system model with unknown parameters and one-operation communication delays, but the accurate system information is required in [19-22].

\section{Convergence Analysis}

In this section, the convergence of approximation discrepancy is discussed in Theorem 4 and the convergences of tracking errors of algorithm DDOILC (27) plus (28) and DDNOILC (30)-(36) are analyzed in Theorems 6 and 7, respectively.

Theorem 4. Assume that the approximation matrix $\mathbf{H}_{k}$ is updated by (27) or (32). Then the system matrix approximation discrepancy matrix $\left\|\Delta \mathbf{H}_{k}\right\|_{2}$ is convergent to a bounded constant as the iteration increases.

Proof. Since the approximation discrepancy $\Delta \mathbf{H}_{k}$ is defined as $\Delta \mathbf{H}_{k}=\mathbf{H}-\mathbf{H}_{k}$, then by (27) or (32), it follows that

$$
\begin{aligned}
\Delta \mathbf{H}_{k} & =\Delta \mathbf{H}_{k-1}-\left(\mathbf{H}_{k}-\mathbf{H}_{k-1}\right) \\
& =\Delta \mathbf{H}_{k-1}-\gamma_{k}\left(y_{k}-\mathbf{H}_{k-1} u_{k}\right) u_{k}^{T} \\
& =\Delta \mathbf{H}_{k-1}-\gamma_{k}\left(\mathbf{H} u_{k}-\mathbf{H}_{k-1} u_{k}\right) u_{k}^{T} \\
& =\Delta \mathbf{H}_{k-1}\left(\mathbf{I}-\gamma_{k} u_{k} u_{k}^{T}\right) .
\end{aligned}
$$

Taking 2-norm on both sides of (37) yields

$$
\left\|\Delta \mathbf{H}_{k}\right\|_{2} \leq\left\|\Delta \mathbf{H}_{k-1}\left(\mathbf{I}-\gamma_{k} u_{k} u_{k}^{T}\right)\right\|_{2} .
$$

In addition, since $\mathbf{I}-\gamma_{k} u_{k} u_{k}^{T}$ is a real symmetric matrix, it yields

$$
\begin{aligned}
\left\|\mathbf{I}-\gamma_{k} u_{k} u_{k}^{T}\right\|_{2}^{2} & =\max _{1 \leq i \leq N}\left\{\left|\lambda_{i}\left(\mathbf{I}-\gamma_{k} u_{k} u_{k}^{T}\right)\right|\right\} \\
& =\max _{1 \leq i \leq N}\left\{\left|1-\gamma_{k} \lambda_{i}\left(u_{k} u_{k}^{T}\right)\right|\right\} \\
& =\max _{1 \leq i \leq N}\left\{\left|1-\frac{\lambda_{i}\left(u_{k} u_{k}^{T}\right)}{\mu+\left\|u_{k}\right\|_{2}^{2}}\right|\right\} \\
& =\max _{1 \leq i \leq N}\left\{1-\frac{\lambda_{i}\left(u_{k} u_{k}^{T}\right)}{\mu+\lambda_{\max }\left(u_{k} u_{k}^{T}\right)}\right\} \\
& =1-\frac{\lambda_{\min }\left(u_{k} u_{k}^{T}\right)}{\mu+\lambda_{\max }\left(u_{k} u_{k}^{T}\right)} .
\end{aligned}
$$


Since $\mu>0$ and $\lambda_{\min }\left(u_{k} u_{k}^{T}\right) \geq 0$, then

$$
0<1-\frac{\lambda_{\min }\left(u_{k} u_{k}^{T}\right)}{\mu+\lambda_{\max }\left(u_{k} u_{k}^{T}\right)} \leq 1 .
$$

According to (38), (39), and (40), it is easy to have

$$
\left\|\Delta \mathbf{H}_{k}\right\|_{2} \leq\left\|\Delta \mathbf{H}_{k-1}\right\|_{2} \text {. }
$$

Hence, the approximation discrepancy consequence $\left\{\left\|\Delta \mathbf{H}_{k}\right\|_{2}\right\}$ is convergent to a bounded constant; that is,

$$
\lim _{k \rightarrow \infty}\left\|\Delta \mathbf{H}_{k}\right\|_{2}=d_{1}
$$

where $d_{1}$ is a bounded constant.

This completes the proof.

Remark 5. It can be seen from (41) that the approximation $\mathbf{H}_{k}$ does not go farther from the system matrix $\mathbf{H}$ than $\mathbf{H}_{k-1}$ along the operation. Inequality (40) implies that if $\lambda_{\min }\left(u_{k} u_{k}^{T}\right)$ is not equal to zero, then the approximation discrepancy $\left\|\Delta \mathbf{H}_{k}\right\|_{2}$ is convergent to zero and the boundary $\xi_{1}$ is equal to zero. Further, if $\lambda_{\min }\left(u_{k} u_{k}^{T}\right)$ is equal to zero, then the approximation discrepancy $\left\|\Delta \mathbf{H}_{k}\right\|_{2}$ is no longer declining. Thus the boundary $\xi_{1}$ is greater than zero. It is worth noting that if $\lambda_{\min }\left(u_{k} u_{k}^{T}\right)$ is very small but not equal to zero, then $\left\|\Delta \mathbf{H}_{k}\right\|_{2}$ declines very slowly.

Theorem 6. Assume that the system matrix $\mathbf{H}$ is nonsingular and communication delays do not occur in NCSs. Then the tracking errors of DDOILC scheme (27) plus (28) are monotonically convergent to zero if the parameter $\varepsilon$ is appropriately chosen.

Proof. From (26) and (28), the tracking error is derived as

$$
\begin{aligned}
e_{k+1} & =y_{d}-y_{k+1}=y_{d}-\mathbf{H} u_{k+1} \\
& =y_{d}-\mathbf{H}\left(u_{k}+\frac{\mathbf{H}_{k}^{T} e_{k}}{\varepsilon+\lambda_{\max }\left(\mathbf{H}_{k} \mathbf{H}_{k}^{T}\right)}\right) \\
& =\left(\mathbf{I}-\frac{\mathbf{H H}_{k}^{T}}{\varepsilon+\lambda_{\max }\left(\mathbf{H}_{k} \mathbf{H}_{k}^{T}\right)}\right) e_{k} .
\end{aligned}
$$

It is noticed that $\Delta \mathbf{H}_{k}=\mathbf{H}-\mathbf{H}_{k}$ yields $\mathbf{H}_{k}=\mathbf{H}-\Delta \mathbf{H}_{k}$. Then,

$$
\begin{aligned}
\mathbf{I} & -\frac{\mathbf{H H}_{k}^{T}}{\varepsilon+\lambda_{\max }\left(\mathbf{H}_{k} \mathbf{H}_{k}^{T}\right)} \\
& =\left(\mathbf{I}-\frac{\mathbf{H} \mathbf{H}^{T}}{\varepsilon+\lambda_{\max }\left(\mathbf{H}_{k} \mathbf{H}_{k}^{T}\right)}\right)+\frac{\mathbf{H}\left(\Delta \mathbf{H}_{k}^{T}\right)}{\varepsilon+\lambda_{\max }\left(\mathbf{H}_{k} \mathbf{H}_{k}^{T}\right)} .
\end{aligned}
$$

According to the concept of limit, expression (42) implies

$$
\left\|\left(\Delta \mathbf{H}_{k}\right)\right\|_{2} \leq \xi_{1}, \quad \forall k=1,2, \ldots,
$$

where $\xi_{1}$ is a bounded constant satisfying $\xi_{1} \geq d_{1}$.
Further, from (45), we have

$$
\begin{aligned}
\|\mathbf{H}\|_{2} & =\left\|\mathbf{H}_{k}+\Delta \mathbf{H}_{k}\right\|_{2} \leq\left\|\mathbf{H}_{k}\right\|_{2}+\left\|\Delta \mathbf{H}_{k}\right\|_{2} \\
& \leq\left\|\mathbf{H}_{k}\right\|_{2}+\xi_{1}=\sqrt{\lambda_{\max }\left(\mathbf{H}_{k} \mathbf{H}_{k}^{T}\right)}+\xi_{1} .
\end{aligned}
$$

By (46), choose appropriate parameter $\varepsilon$ such that

$$
\begin{aligned}
\frac{\left\|\mathbf{H}\left(\Delta \mathbf{H}_{k}^{T}\right)\right\|_{2}}{\varepsilon+\lambda_{\max }\left(\mathbf{H}_{k} \mathbf{H}_{k}^{T}\right)} & \leq \frac{\|\mathbf{H}\|_{2}}{\varepsilon+\lambda_{\max }\left(\mathbf{H}_{k} \mathbf{H}_{k}^{T}\right)}\left\|\Delta \mathbf{H}_{k}^{T}\right\|_{2} \\
& \leq \frac{\sqrt{\lambda_{\max }\left(\mathbf{H}_{k} \mathbf{H}_{k}^{T}\right)}+\xi_{1}}{\varepsilon+\lambda_{\max }\left(\mathbf{H}_{k} \mathbf{H}_{k}^{T}\right)} \xi_{1}<\xi_{2},
\end{aligned}
$$

where $\xi_{2}$ is a positive constant and $0<\xi_{2}<1$.

Since $\mathbf{H}$ is nonsingular, then the matrix $\mathbf{H H}^{T}$ is symmetrical and positively definite. Therefore, there exist an orthogonal matrix $\mathbf{Q}$ such that

$$
\mathbf{H} \mathbf{H}^{T}=\mathbf{Q}^{-1} \Lambda \mathbf{Q},
$$

where $\boldsymbol{\Lambda}=\operatorname{diag}\left[\lambda_{1}, \lambda_{2}, \ldots, \lambda_{N}\right]$ with $\left\{\lambda_{1}, \lambda_{2}, \ldots, \lambda_{N}\right\}$ being positive eigenvalues of matrix $\mathbf{H H}^{T}$. Thus, there exists an appropriate parameter $\varepsilon$ such that

$$
\begin{aligned}
\| \mathbf{I} & -\frac{\mathbf{H H}^{T}}{\varepsilon+\lambda_{\max }\left(\mathbf{H}_{k} \mathbf{H}_{k}^{T}\right)} \|_{2} \\
& =\left\|\mathbf{Q}^{-1}\left(\mathbf{I}-\frac{\boldsymbol{\Lambda}}{\varepsilon+\lambda_{\max }\left(\mathbf{H}_{k} \mathbf{H}_{k}^{T}\right)}\right) \mathbf{Q}\right\|_{2}<\tau-\xi_{2} \\
& <1,
\end{aligned}
$$

where $\tau$ is a positive constant which is less than unity.

Therefore, it follows from (47) and (49) that

$$
\begin{aligned}
\| \mathbf{I} & -\frac{\mathbf{H H}_{k}^{T}}{\varepsilon+\lambda_{\max }\left(\mathbf{H}_{k} \mathbf{H}_{k}^{T}\right)} \|_{2} \\
& \leq\left\|\mathbf{I}-\frac{\mathbf{H H}^{T}}{\varepsilon+\lambda_{\max }\left(\mathbf{H}_{k} \mathbf{H}_{k}^{T}\right)}\right\|_{2}+\frac{\left\|\mathbf{H}\left(\Delta \mathbf{H}_{k}^{T}\right)\right\|_{2}}{\varepsilon+\lambda_{\max }\left(\mathbf{H}_{k} \mathbf{H}_{k}^{T}\right)} \\
& <\tau<1 .
\end{aligned}
$$

Consequently, inequality (50) results in

$$
\left\|e_{k+1}\right\|_{2} \leq \tau\left\|e_{k}\right\|_{2} \leq \tau^{2}\left\|e_{k-1}\right\|_{2} \leq \cdots \leq \tau^{k}\left\|e_{1}\right\|_{2} .
$$

Thus,

$$
\lim _{k \rightarrow \infty}\left\|e_{k+1}\right\|_{2}=0
$$

This completes the proof.

Theorem 7. Assume that the system matrix $\mathbf{H}$ is nonsingular and the DDNOILC algorithm (30)-(36) is applied to system (9). Then the expectation of tracking error $E\left\{\widetilde{e}_{k+1}\right\}$ is convergent to zero if the parameter $\varepsilon$ is properly selected and the prior probabilities $a$ and $b$ are appropriately given. 
Proof. Denote $\Delta \tilde{u}_{k}=u_{d}-\tilde{u}_{k}, e_{k}=y_{d}-y_{k}$, and $\tilde{e}_{k}=y_{d}-\tilde{y}_{k}$. From (9), (11), and (30), it yields

$$
\begin{aligned}
e_{k} & =y_{d}-y_{k}=y_{d}-\mathbf{H} u_{k}=y_{d}-\mathbf{H}\left(\boldsymbol{\Psi}_{k} \tilde{u}_{k}+\bar{\Psi}_{k} \tilde{u}_{k-1}\right) \\
& =\mathbf{H} \Psi_{k} \Delta \tilde{u}_{k}+\mathbf{H} \bar{\Psi}_{k} \Delta \tilde{u}_{k-1} .
\end{aligned}
$$

Meanwhile, by (33), it is easy to get

$$
\begin{aligned}
\tilde{e}_{k} & =y_{d}-\tilde{y}_{k}=y_{d}-\left(\boldsymbol{\Phi}_{k} y_{k}+\overline{\boldsymbol{\Phi}}_{k} y_{k-1}\right) \\
& =\boldsymbol{\Phi}_{k} e_{k}+\overline{\boldsymbol{\Phi}}_{k} e_{k-1} .
\end{aligned}
$$

From (35), (36), (53), and (54), we have

$$
\begin{aligned}
& \Delta \widetilde{u}_{k+1}=u_{d}-\widetilde{u}_{k+1}=u_{d}-\left(\widetilde{u}_{k}+\pi_{k} \mathbf{H}_{k}^{T} \widetilde{e}_{k}\right)=u_{d}-\widetilde{u}_{k} \\
& -\pi_{k} \mathbf{H}_{k}^{T}\left(\Phi_{k} e_{k}+\bar{\Phi}_{k} e_{k-1}\right)=\Delta \widetilde{u}_{k} \\
& -\frac{\mathbf{H}_{k}^{T}}{\varepsilon+\lambda_{\max }\left(\mathbf{H}_{k} \mathbf{H}_{k}^{T}\right)} \boldsymbol{\Phi}_{k}\left(\mathbf{H} \Psi_{k} \Delta \tilde{u}_{k}+\mathbf{H} \bar{\Psi}_{k} \Delta \tilde{u}_{k-1}\right) \\
& -\frac{\mathbf{H}_{k}^{T}}{\varepsilon+\lambda_{\max }\left(\mathbf{H}_{k} \mathbf{H}_{k}^{T}\right)} \\
& \cdot \overline{\boldsymbol{\Phi}}_{k}\left(\mathbf{H} \Psi_{k-1} \Delta \tilde{u}_{k-1}+\mathbf{H} \bar{\Psi}_{k-1} \Delta \tilde{u}_{k-2}\right) \\
& =\left(\mathbf{I}-\frac{\mathbf{H}_{k}^{T} \boldsymbol{\Phi}_{k} \mathbf{H} \Psi_{k}}{\varepsilon+\lambda_{\max }\left(\mathbf{H}_{k} \mathbf{H}_{k}^{T}\right)}\right) \Delta \widetilde{u}_{k} \\
& -\left(\frac{\mathbf{H}_{k}^{T} \boldsymbol{\Phi}_{k} \mathbf{H} \bar{\Psi}_{k}}{\varepsilon+\lambda_{\max }\left(\mathbf{H}_{k} \mathbf{H}_{k}^{T}\right)}+\frac{\mathbf{H}_{k}^{T} \overline{\boldsymbol{\Phi}}_{k} \mathbf{H} \Psi_{k-1}}{\varepsilon+\lambda_{\max }\left(\mathbf{H}_{k} \mathbf{H}_{k}^{T}\right)}\right) \Delta \widetilde{u}_{k-1} \\
& -\left(\frac{\mathbf{H}_{k}^{T} \overline{\boldsymbol{\Phi}}_{k} \mathbf{H} \bar{\Psi}_{k-1}}{\varepsilon+\lambda_{\max }\left(\mathbf{H}_{k} \mathbf{H}_{k}^{T}\right)}\right) \Delta \widetilde{u}_{k-2} .
\end{aligned}
$$

$$
\begin{aligned}
& E\left\{\overline{\boldsymbol{\Phi}}_{k} \boldsymbol{\Psi}_{k-1}\right\}=E\left\{\overline{\boldsymbol{\Phi}}_{k}\right\} E\left\{\boldsymbol{\Psi}_{k-1}\right\}=a \bar{b} \mathbf{I}, \\
& E\left\{\overline{\boldsymbol{\Phi}}_{k} \overline{\boldsymbol{\Psi}}_{k-1}\right\}=E\left\{\overline{\boldsymbol{\Phi}}_{k}\right\} E\left\{\overline{\boldsymbol{\Psi}}_{k-1}\right\}=\bar{a} \bar{b} \mathbf{I},
\end{aligned}
$$

where $\bar{a}=1-a$ and $\bar{b}=1-b$.

Calculating mathematical expectation on both sides of (55) obtains

$$
\begin{aligned}
E\left\{\Delta \tilde{u}_{k+1}\right\}= & \left(\mathbf{I}-\frac{a b \mathbf{H}_{k}^{T} \mathbf{H}}{\varepsilon+\lambda_{\max }\left(\mathbf{H}_{k} \mathbf{H}_{k}^{T}\right)}\right) E\left\{\Delta \tilde{u}_{k}\right\} \\
& -\left(\frac{(\bar{a} b+a \bar{b}) \mathbf{H}_{k}^{T} \mathbf{H}}{\varepsilon+\lambda_{\max }\left(\mathbf{H}_{k} \mathbf{H}_{k}^{T}\right)}\right) E\left\{\Delta \widetilde{u}_{k-1}\right\} \\
& -\left(\frac{\bar{a} \bar{b} \mathbf{H}_{k}^{T} \mathbf{H}}{\varepsilon+\lambda_{\max }\left(\mathbf{H}_{k} \mathbf{H}_{k}^{T}\right)}\right) E\left\{\Delta \tilde{u}_{k-2}\right\} .
\end{aligned}
$$

Taking 2-norm in both sides of (57) reduces

$$
\begin{aligned}
\left\|E\left\{\Delta \tilde{u}_{k+1}\right\}\right\|_{2} \leq & \delta_{k}^{1}\left\|E\left\{\Delta \tilde{u}_{k}\right\}\right\|_{2}+\delta_{k}^{2}\left\|E\left\{\Delta \tilde{u}_{k-1}\right\}\right\|_{2} \\
& +\delta_{k}^{3}\left\|E\left\{\Delta \tilde{u}_{k-2}\right\}\right\|_{2},
\end{aligned}
$$

where

$$
\begin{aligned}
& \delta_{k}^{1}=\left\|\mathbf{I}-\frac{a b \mathbf{H}_{k}^{T} \mathbf{H}}{\varepsilon+\lambda_{\max }\left(\mathbf{H}_{k} \mathbf{H}_{k}^{T}\right)}\right\|_{2}, \\
& \delta_{k}^{2}=\frac{\left\|(\bar{a} b+a \bar{b}) \mathbf{H}_{k}^{T} \mathbf{H}\right\|}{\varepsilon+\lambda_{\max }\left(\mathbf{H}_{k} \mathbf{H}_{k}^{T}\right)}, \\
& \delta_{k}^{3}=\frac{\left\|\bar{a} \bar{b} \mathbf{H}_{k}^{T} \mathbf{H}\right\|_{2}}{\varepsilon+\lambda_{\max }\left(\mathbf{H}_{k} \mathbf{H}_{k}^{T}\right)} .
\end{aligned}
$$

Then

$$
\begin{aligned}
\delta_{k}^{1} \leq & \left\|\mathbf{I}-\frac{a b \mathbf{H}^{T} \mathbf{H}}{\varepsilon+\lambda_{\max }\left(\mathbf{H}_{k} \mathbf{H}_{k}^{T}\right)}\right\|_{2}+\frac{a b\left\|\left(\Delta \mathbf{H}_{k}^{T}\right) \mathbf{H}\right\|_{2}}{\varepsilon+\lambda_{\max }\left(\mathbf{H}_{k} \mathbf{H}_{k}^{T}\right)} \\
= & \left\|\mathbf{Q}^{-1}\left(\mathbf{I}-\frac{a b \mathbf{\Lambda}}{\varepsilon+\lambda_{\max }\left(\mathbf{H}_{k} \mathbf{H}_{k}^{T}\right)}\right) \mathbf{Q}\right\|_{2} \\
& +\frac{a b\left\|\left(\Delta \mathbf{H}_{k}^{T}\right) \mathbf{H}\right\|_{2}}{\varepsilon+\lambda_{\max }\left(\mathbf{H}_{k} \mathbf{H}_{k}^{T}\right)}, \\
\delta_{k}^{2} \leq & \frac{\|(\bar{a} b+a \bar{b}) \mathbf{H}^{T} \mathbf{H}_{2}}{\varepsilon+\lambda_{\max }\left(\mathbf{H}_{k} \mathbf{H}_{k}^{T}\right)}+\frac{(\bar{a} b+a \bar{b}) \|\left(\Delta \mathbf{H}_{k}\right)^{T} \mathbf{H}_{2}}{\varepsilon+\lambda_{\max }\left(\mathbf{H}_{k} \mathbf{H}_{k}^{T}\right)} \\
\leq & \frac{(\bar{a} b+a \bar{b})\left\|\mathbf{Q}^{-1} \Lambda \mathbf{Q}\right\|_{2}}{\varepsilon+\lambda_{\max }\left(\mathbf{H}_{k} \mathbf{H}_{k}^{T}\right)} \\
\delta_{k}^{3} \leq & \frac{(\bar{a} b+a \bar{b})\left\|\left(\Delta \mathbf{H}_{k}\right)^{T} \mathbf{H}\right\|_{2}}{\varepsilon+\lambda_{\max }\left(\mathbf{H}_{k} \mathbf{H}_{k}^{T}\right)}+\frac{\bar{\varepsilon} \bar{b} \|\left(\Delta \mathbf{H}_{k}\right)^{T} \mathbf{H}_{2}}{\varepsilon+\lambda_{\max }\left(\mathbf{H}_{k} \mathbf{H}_{k}^{T}\right)} . \\
& \left.+\mathbf{H}_{k} \mathbf{H}_{k}^{T}\right)
\end{aligned}
$$

Thus, it can be derived as

$$
\begin{aligned}
\delta_{k}^{1}+\delta_{k}^{2}+\delta_{k}^{3} \leq & \left\|\mathbf{Q}^{-1}\left(\mathbf{I}-\frac{a b \boldsymbol{\Lambda}}{\varepsilon+\lambda_{\max }\left(\mathbf{H}_{k} \mathbf{H}_{k}^{T}\right)}\right) \mathbf{Q}\right\|_{2} \\
& +\frac{(1-a b)\left\|\mathbf{Q}^{-1} \boldsymbol{\Lambda} \mathbf{Q}\right\|_{2}}{\varepsilon+\lambda_{\max }\left(\mathbf{H}_{k} \mathbf{H}_{k}^{T}\right)} \\
& +\frac{\|\mathbf{H}\|_{2}}{\varepsilon+\lambda_{\max }\left(\mathbf{H}_{k} \mathbf{H}_{k}^{T}\right)}\left\|\Delta \mathbf{H}_{k}^{T}\right\|_{2} .
\end{aligned}
$$

According to (45) and (46), choosing appropriate parameter $\varepsilon$ satisfies 


$$
\frac{\|\mathbf{H}\|_{2}}{\varepsilon+\lambda_{\max }\left(\mathbf{H}_{k} \mathbf{H}_{k}^{T}\right)}\left\|\Delta \mathbf{H}_{k}^{T}\right\|_{2}<\xi_{3},
$$

where $\xi_{3}$ is a positive constant satisfying $0<\xi_{3}<1$.

Since all the eigenvalues $\left\{\lambda_{1}, \lambda_{2}, \ldots, \lambda_{N}\right\}$ of matrix $\mathbf{H H}^{T}$ are positive, there exists an appropriate parameter $\varepsilon$ such that

$$
\begin{gathered}
\left\|\mathbf{Q}^{-1}\left(\mathbf{I}-\frac{a b}{\varepsilon+\lambda_{\max }\left(\mathbf{H}_{k} \mathbf{H}_{k}^{T}\right)} \boldsymbol{\Lambda}\right) \mathbf{Q}\right\|_{2} \\
+\frac{(1-a b)\left\|\mathbf{Q}^{-1} \Lambda \mathbf{Q}\right\|_{2}}{\varepsilon+\lambda_{\max }\left(\mathbf{H}_{k} \mathbf{H}_{k}^{T}\right)}<\delta-\xi_{3}<1,
\end{gathered}
$$

where $0<\delta<1$.

Thus, from (61), (62), and (63), we have

$$
\delta_{k}^{1}+\delta_{k}^{2}+\delta_{k}^{3}<\delta<1 .
$$

By (58) and (64), it has

$$
\begin{gathered}
\left\|E\left\{\Delta \tilde{u}_{3 k+l}\right\}\right\|_{2} \leq \delta \max \left\{\left\|E\left\{\Delta \tilde{u}_{3 k}\right\}\right\|_{2},\left\|E\left\{\Delta \tilde{u}_{3 k-1}\right\}\right\|_{2},\right. \\
\left.\left\|E\left\{\Delta \tilde{u}_{3 k-2}\right\}\right\|_{2}\right\} \leq \delta^{2} \max \left\{\left\|E\left\{\Delta \tilde{u}_{3 k-3}\right\}\right\|_{2},\right. \\
\left.\left\|E\left\{\Delta \tilde{u}_{3 k-4}\right\}\right\|_{2},\left\|E\left\{\Delta \tilde{u}_{3 k-5}\right\}\right\|_{2}\right\} \\
\vdots \\
\leq \delta^{k} \max \left\{\left\|E\left\{\Delta \widetilde{u}_{3}\right\}\right\|_{2},\left\|E\left\{\Delta \widetilde{u}_{2}\right\}\right\|_{2},\left\|E\left\{\Delta \widetilde{u}_{1}\right\}\right\|_{2}\right\},
\end{gathered}
$$

where $l=1,2,3$.

Then,

$$
\lim _{k \rightarrow \infty}\left\|E\left\{\Delta \tilde{u}_{3 k+l}\right\}\right\|_{2}=0, \quad l=1,2,3 .
$$

According to the concept of limit, we have

$$
\lim _{k \rightarrow \infty}\left\|E\left\{\Delta \tilde{u}_{k+1}\right\}\right\|_{2}=0 .
$$

From (53), (54), and (55), it is easy to deduce that

$$
\begin{aligned}
E\left\{\widetilde{e}_{k+1}\right\}= & (a b \mathbf{H}) E\left\{\Delta \widetilde{u}_{k+1}\right\}+(\bar{a} b+a \bar{b}) \mathbf{H} E\left\{\Delta \widetilde{u}_{k}\right\} \\
& +\bar{a} \bar{b} \mathbf{H} E\left\{\Delta \widetilde{u}_{k-1}\right\} .
\end{aligned}
$$

Taking 2-norm on both sides of (68), we have

$$
\begin{aligned}
\left\|E\left\{\widetilde{e}_{k+1}\right\}\right\|_{2} \leq & a b\|\mathbf{H}\|_{2}\left\|E\left\{\Delta \tilde{u}_{k+1}\right\}\right\|_{2} \\
& +(\bar{a} b+a \bar{b})\|\mathbf{H}\|_{2}\left\|E\left\{\Delta \tilde{u}_{k}\right\}\right\|_{2} \\
& +\bar{a} \bar{b}\|\mathbf{H}\|_{2}\left\|E\left\{\Delta \tilde{u}_{k-1}\right\}\right\|_{2} .
\end{aligned}
$$

Since the matrix $\mathbf{H}$ is bounded. By (68) and (69), we obtain the conclusion

$$
\lim _{k \rightarrow 0}\left\|E\left\{\widetilde{e}_{k+1}\right\}\right\|_{2}=0
$$

This completes the proof.
Remark 8. Notice that Theorem 7 displays that the tracking errors of DDNOILC algorithm converge to zero when the one-operation compensation approaches (3) and (5) are applied into system (9). In [21, 22], one-step compensation approach has been involved and the tracking errors have been proved to be bounded. Thus, the presented DDNOILC in this paper is effective. The detailed comparisons between one operation and one step are demonstrated in the next section.

\section{Numerical Simulations}

In microelectronics manufacturing, the rapid thermal processing is regarded as a repetitive batch process [23]. The ILC scheme is adequately to be utilized so that the transient temperature of the reactor to follow a desired trajectory. Suppose that the transfer function of the reactor is identified as $G_{p}(s)=K /\left(\left(\tau_{W} s+1\right)\left(\tau_{L} s+1\right)\right)$, where $K$ is the process gain, $\tau_{W}$ denotes the heating time constant of the crystal, and $\tau_{L}$ denotes the heating time constant of the crystal light. Conventionally, the power ratio of the crystal light is tuned by a proportional-derivative-integral (PID) controller. Given that the transfer function of the PID controller is $G_{C}(s)=$ $K_{C} /\left(1+1 / \tau_{I} s+\tau_{D} s\right)$, where $K_{C}, \tau_{I}$, and $\tau_{D}$ are proportional, integral, and derivative gains, respectively. By converting the dynamics of frequency domain into that of time domain and then discretizing the PID-controller-tuned closed-loop control system with the sampling step $\Delta t=0.005$, the discrete-time system is described as follows:

$$
\begin{aligned}
x_{k}(t+1)= & {\left[\begin{array}{ccc}
1 & \Delta t & 0 \\
0 & 1 & \Delta t \\
-\Delta t a_{0} & -\Delta t a_{1} & 1-\Delta t a_{2}
\end{array}\right] x_{k}(t) } \\
& +\left[\begin{array}{c}
0 \\
0 \\
\Delta t
\end{array}\right] u_{k}(t), \\
y_{k}(t)= & {\left[\begin{array}{lll}
b_{0} & b_{1} & b_{2}
\end{array}\right] x_{k}(t), }
\end{aligned}
$$

where

$$
\begin{aligned}
& a_{0}=\frac{K K_{C}}{\tau_{I} \tau_{W} \tau_{L}}, \\
& a_{1}=\frac{1+K K_{C}}{\tau_{W} \tau_{L}}, \\
& a_{2}=\frac{\left(\tau_{W}+\tau_{L}\right)+K K_{C} \tau_{D}}{\tau_{W} \tau_{L}}, \\
& b_{0}=\frac{K K_{C}}{\tau_{I} \tau_{W} \tau_{L}}, \\
& b_{1}=\frac{K K_{C}}{\tau_{W} \tau_{L}}, \\
& b_{2}=\frac{K K_{C} \tau_{D}}{\tau_{W} \tau_{L}} .
\end{aligned}
$$


Set the parameters as $K=22, \tau_{W}=5, \tau_{L}=1.6, K_{C}=24$, $\tau_{I}=5$, and $\tau_{D}=2.83-\exp (-2 t)$ which is time-varying.

The desired trajectory is defined as $y_{d}(t)=1-\exp (-0.2 t)$, $t \in\{0, \ldots, 200\}$. Set the initial states as $x_{k}(0)=\left[\begin{array}{lll}0 & 0 & 0\end{array}\right]^{T}, k=$ $1,2, \ldots, 30$. The beginning control input is chosen as $u_{1}(t)=$ 0 .

Select a group parameters as $\varepsilon=1.5$ and $\mu=150$ such that convergent conditions (50) and (64) are satisfied under the given $\mathbf{H}_{1}$.

In terms of mathematical expectation, the simulations are made for 100 runs, where the notion "one run" means that the proposed ILC scheme is in processing until the perfect tracking is achieved. Thus, $E\left\{y_{k}(t)\right\}$ and $E\left\{\widetilde{e}_{k}\right\}$ are computed as

$$
\begin{gathered}
E\left\{y_{k}(t)\right\}=\frac{1}{100} \sum_{m=1}^{100} y_{k}^{(m)}(t), \\
E\left\{\widetilde{e}_{k}\right\}=\frac{1}{100} \sum_{m=1}^{100} \widetilde{e}_{k}^{(m)},
\end{gathered}
$$

where the superscript $m$ stands for the index of the experiment runs.

In [16], for ensuring the convergence of the presented ILC algorithm, the approximation matrix is required to be diagonally dominant such that

$$
\begin{aligned}
\left|h_{k}^{i j}\right| \leq c_{1}, \quad i \neq j, \quad i=1, \ldots, N, \quad j=1, \ldots, N, & \\
c_{2} & \leq\left|h_{k}^{i i}\right| \leq q c_{2}, \quad q \geq 1, \quad c_{2}>c_{1}(2 q+1)(N-1),
\end{aligned}
$$

where $c_{1}$ and $c_{2}$ are positive constants. For comparison with DDOILC and DDNOILC, the algorithms in [16] are denoted as DD-DDOILC and DD-DDNOILC, respectively.

In [21, 22], one-step communication delays are discussed as follows:

$$
\begin{aligned}
& u_{k}(t)=\alpha_{k}(t) \tilde{u}_{k}(t)+\left(1-\alpha_{k}(t)\right) \widetilde{u}_{k}(t-1), \\
& \tilde{y}_{k}(t)=\beta_{k}(t) y_{k}(t)+\left(1-\beta_{k}(t)\right) y_{k}(t-1) .
\end{aligned}
$$

For comparison with DDNOILC, the algorithm in $[21,22]$ is abbreviated as OS-DDNOILC.

In this section, the approximation behavior and tracking performance of the DDOILC and DDNOILC are simulated by the following cases.

Case 1 (set $a=1$ and $b=1)$. Let

$$
\mathbf{H}_{1}=\left(h_{1}{ }^{i j}\right)_{N \times N}= \begin{cases}1, & i=j, \\ 0.004, & \text { else. }\end{cases}
$$

This case means that no communication delays occur in Figure 1; namely, the OS-DDNOILC is no other than the DDOILC. Thus, it is testified that convergent condition (50) of the proposed DDOILC is guaranteed by setting $\tau=0.98$. It is observed that, for the given matrix $\mathbf{H}_{1}$, the parameters in (74) may be chosen as $c_{1} \geq 0.004, c_{2} \leq 1$, and $q=1$. But they break the inequality $c_{2}>c_{1}(2 q+1)(N-1)$. This implies that

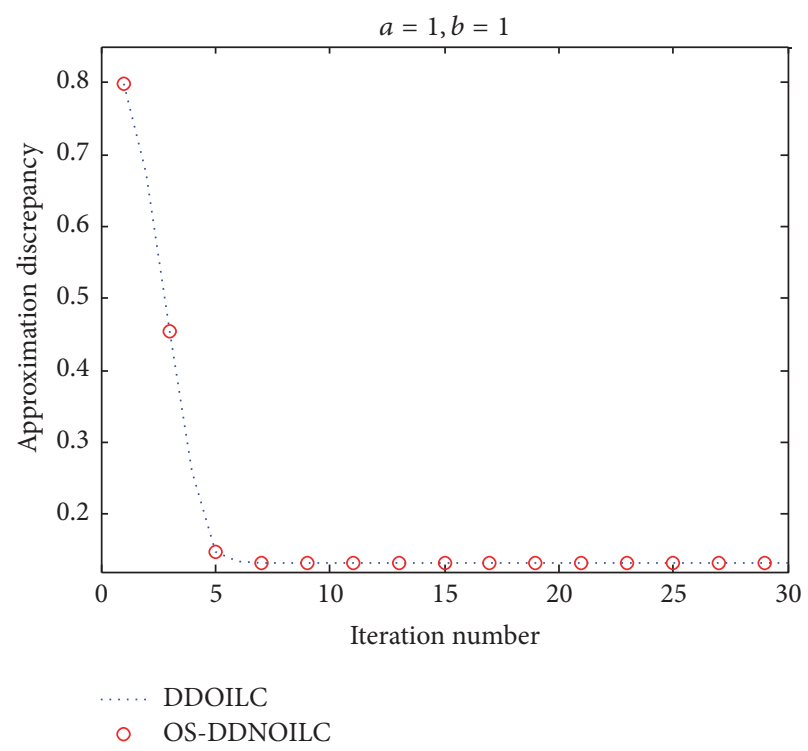

FIgURE 2: Approximation discrepancy of DDOILC and OSDDNOILC.

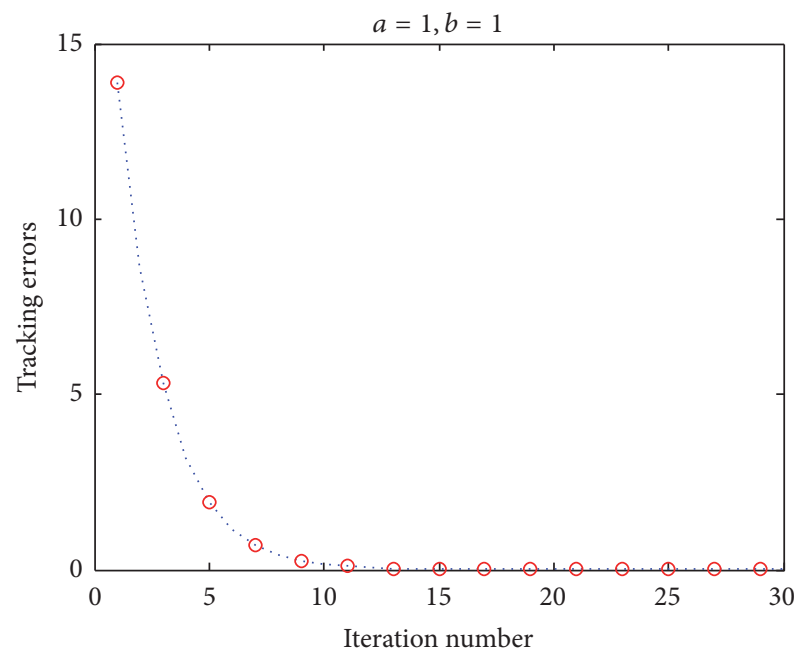

DDOILC

- OS-DDNOILC

FIGURE 3: Tracking errors convergences of DDOILC and OSDDNOILC.

the given matrix $\mathbf{H}_{1}$ does not satisfy the convergent condition of the DD-DDOILC in [16].

Figure 2 displays that the convergence of the approximation discrepancy of DDOILC and OS-DDNOILC is descending but bounded, whilst Figure 3 exhibits that the tracking errors of both the DDOILC and the OS-DDNOILC are monotonously convergent to a bounded constant. This conveys that the convergent condition (74) for the existing DD-DDOILC algorithm is sufficient but not necessary.

Figure 4 demonstrates the outputs of DDOILC at the 5th and 10th iterations, respectively, where the dash curve refers 


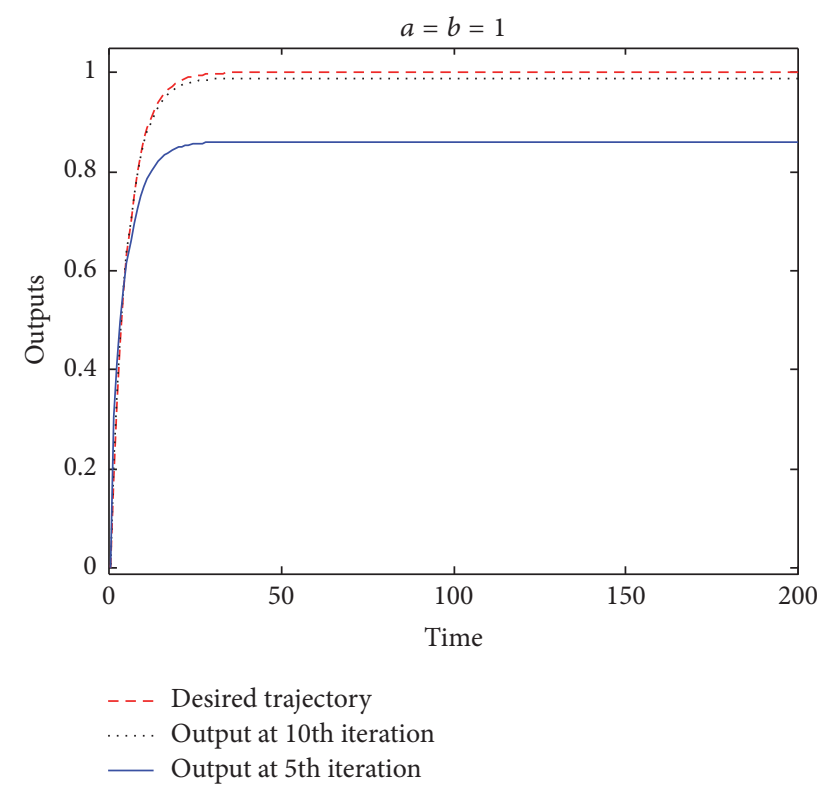

FIGURE 4: Outputs of DDOILC.

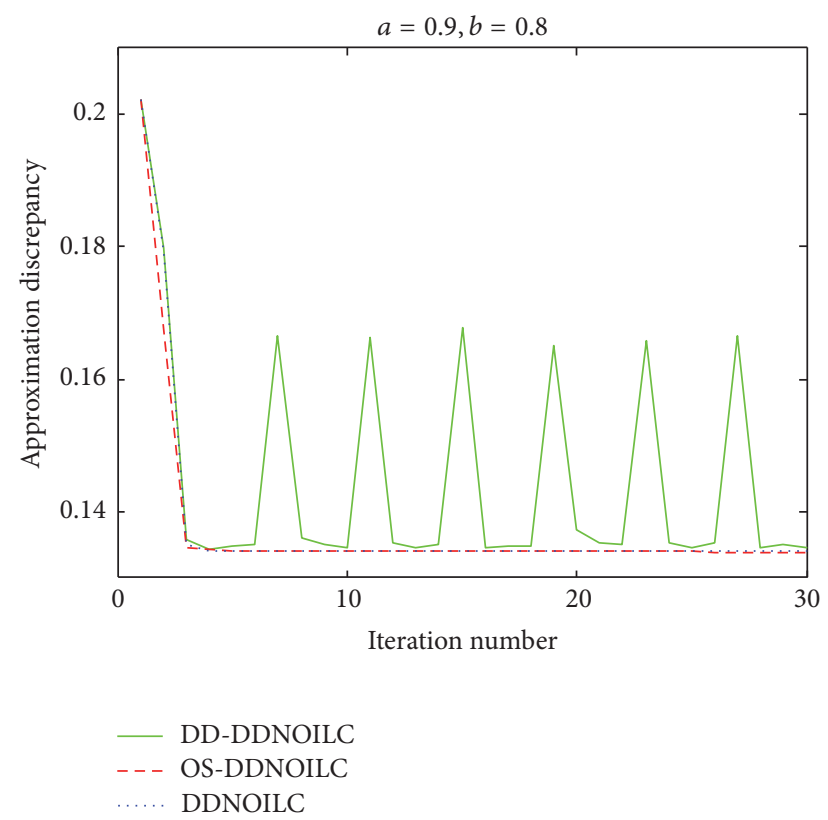

FIGURE 5: Approximation discrepancy of DD-DDNOILC, OSDDNOILC, and DDNOILC schemes.

to the desired trajectory, the solid curve plots the output at the 5 th iteration, and the dotted one shows the output at the 10 th iteration, respectively.

Case 2 (set $a=0.9$ and $b=0.8$ ). Let

$$
\mathbf{H}_{1}=\left(h_{1}{ }^{i j}\right)= \begin{cases}1, & i=j \\ 0.001, & \text { else. }\end{cases}
$$

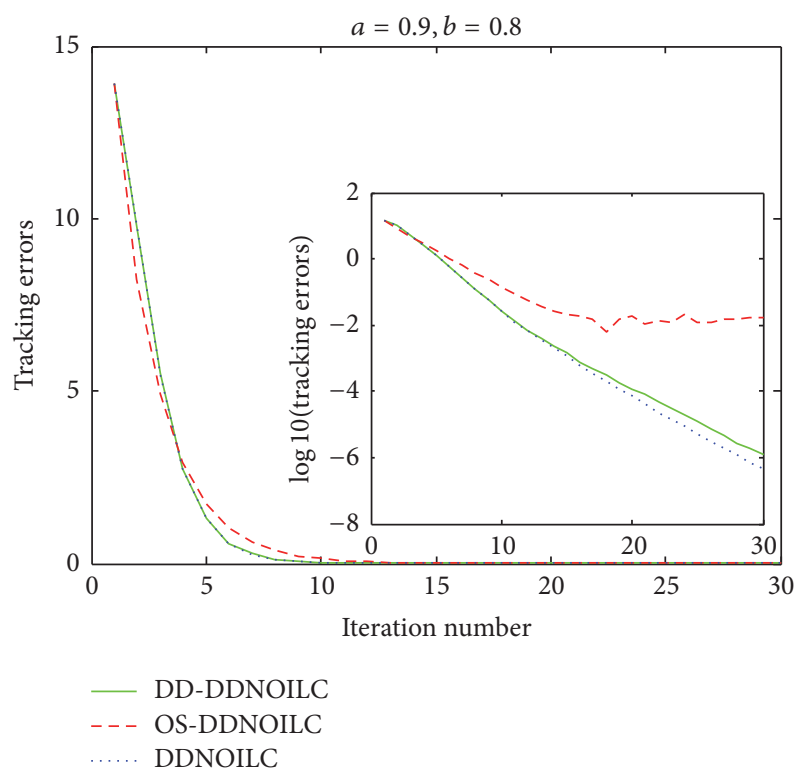

Figure 6: Tracking errors of DD-DDNOILC, OS-DDNILC, and DDNOILC.

This case implies that the one-operation communication delays occur in Figure 1. It is computed that convergent condition (64) of the proposed DDNOILC is satisfied by setting $\delta=0.981$. Meanwhile, all the inequalities in formulation (74) are ensured by selecting $c_{1}=0.001, c_{2}=1$, and $q=1$. This implies that the convergent conditions for both the proposed DDNOILC and the DD-DDNOILC are guaranteed.

Figure 5 manifests the convergences of the approximation discrepancy of DDNOILC, DD-DDNOILC, and OSDDNOILC, respectively, which conveys that the approximation discrepancies $\left\|\Delta \mathbf{H}_{k}\right\|_{2}$ are bounded. However, it is seen that the approximation discrepancies of DDNOILC and OS-DDOILC are monotonously convergent to a bounded constant but that of the DD-DDNOILC is oscillatory with higher magnitude.

Figure 6 gives the convergences of the tracking errors of DD-DDNOILC, OS-DDNOILC, and DDNOILC measured in the forms of $\left\|E\left\{\widetilde{e}_{k}\right\}\right\|_{2}$ and $\log 10\left(\left\|E\left\{\widetilde{e}_{k}\right\}\right\|_{2}\right)$, respectively, where $\log 10(\cdot)$ is the base-10 logarithm of $(\cdot)$. It is observed that the tracking error of the proposed DDNOILC is slightly less than that of the DD-DDNOILC, but the tracking error of the OS-DDNOILC is bounded.

Figure 7 exhibits the outputs of DDNOILC at the 5th and 10th iterations, respectively.

\section{Conclusion}

In this paper, a kind of data-driven optimal ILC (DDOILC) scheme is constructed for a class of discrete linear tinevarying systems. The scheme generates two sequences of system matrix approximations and upgraded control inputs in an interacted mode by solving sequential iteration-varying minimization problems. The paper achieves not only the faster convergence of the tracking error, but also the perfect 


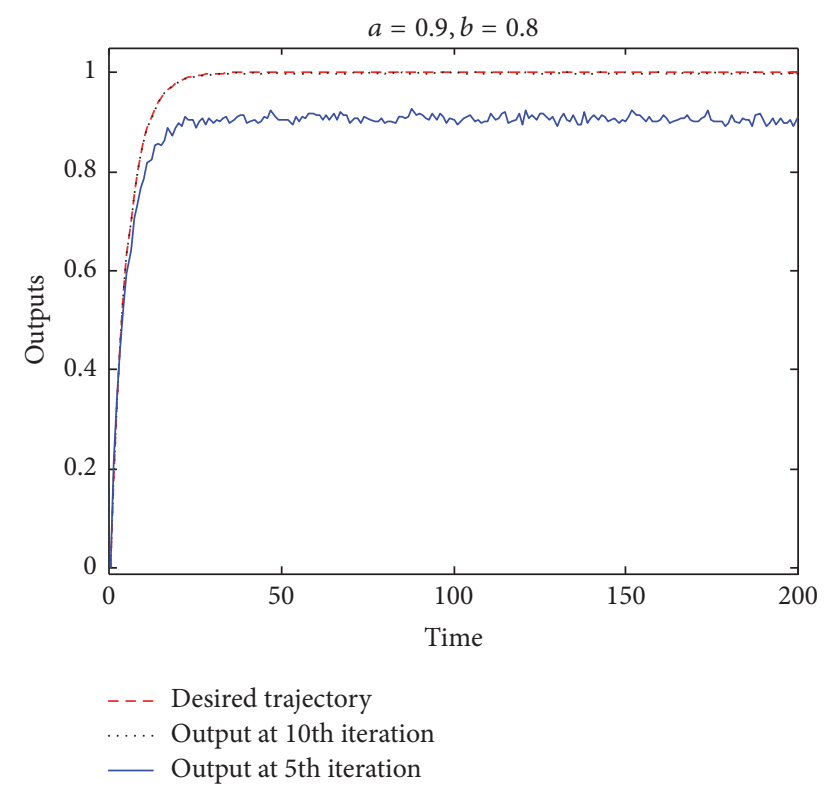

Figure 7: Outputs of DDNOILC.

approximation of the system matrix. The approximation processing may be to some extent regarded as a kind of system identification. This owes to the efficacious harness of the multioperation inputs and outputs. Further, the paper presents a data-driven networked optimal ILC (DDNOILC) strategy for the system with one-operation communication delays. Benefited from the multioperation feature of ILC-driven systems, the strategy relaxes the communication delays from one-step sampling time interval to one-iteration period. As the mending scheme for the delayed data is synchronous for two adjacent iterations, the zero-error convergence is guaranteed. This turns to be the significance of the ILC mechanism. However, how to investigate the tracking behavior of DDOILC and/or DDNOILC for discrete stochastic nonlinear systems with one-operation communication delays is challenging.

\section{Competing Interests}

The authors declare that there is no conflict of interests regarding the publication of this article.

\section{Acknowledgments}

This work was supported by the National Natural Science Foundation of China under Grants F010114-60974140 and 61273135 and the National Research Foundation Korea Grant NRF-2013R1A2A2A01067449.

\section{References}

[1] S. Arimoto, S. Kawamura, and F. Miyazaki, "Bettering operation of Robots by learning," Journal of Robotic Systems, vol. 1, no. 2, pp. 123-140, 1984.
[2] S. S. Saab, "A discrete-time stochastic learning control algorithm," IEEE Transactions on Automatic Control, vol. 46, no. 6, pp. 877-887, 2001.

[3] H.-S. Ahn, Y. Q. Chen, and K. L. Moore, "Iterative learning control: brief survey and categorization," IEEE Transactions on Systems, Man and Cybernetics Part C: Applications and Reviews, vol. 37, no. 6, pp. 1099-1121, 2007.

[4] X. Yang, "A PD-type iterative learning control for a class of switched discrete-time systems with model uncertainties and external noises," Discrete Dynamics in Nature and Society, vol. 2015, Article ID 410292, 11 pages, 2015.

[5] J.-X. Xu and Y. Tan, "Robust optimal design and convergence properties analysis of iterative learning control approaches," Automatica, vol. 38, no. 11, pp. 1867-1880, 2002.

[6] K.-H. Park and Z. Bien, "Intervalized iterative learning control for monotonic convergence in the sense of sup-norm," International Journal of Control, vol. 78, no. 15, pp. 1218-1227, 2005.

[7] X. Ruan, Z. Z. Bien, and Q. Wang, "Convergence characteristics of proportional-type iterative learning control in the sense of Lebesgue- $p$ norm," IET Control Theory and Applications, vol. 6, no. 5, pp. 707-714, 2012.

[8] Y. Fang and T. W. S. Chow, "2-D analysis for iterative learning controller for discrete-time systems with variable initial conditions," IEEE Transactions on Circuits and Systems I: Fundamental Theory and Applications, vol. 50, no. 5, pp. 722-727, 2003.

[9] Z. Qu and J.-X. Xu, "Model-based learning controls and their comparisons using Lyapunov direct method," Asian Journal of Control, vol. 4, no. 1, pp. 99-110, 2002.

[10] B. Xuhui, Z. Hongwei, S. YunZhong, and Y. Fashan, " $H_{\infty}$ ILC design for discrete linear systems with packet dropouts and iteration-varying disturbances," Discrete Dynamics in Nature and Society, vol. 2014, Article ID 587323, 10 pages, 2014.

[11] C. Yin, J.-X. Xu, and Z. Hou, "A high-order internal model based iterative learning control scheme for nonlinear systems with time-iteration-varying parameters," IEEE Transactions on Automatic Control, vol. 55, no. 11, pp. 2665-2670, 2010.

[12] T. Liu, X. Z. Wang, and J. Chen, "Robust PID based indirect-type iterative learning control for batch processes with time-varying uncertainties," Journal of Process Control, vol. 24, no. 12, pp. 95106, 2014.

[13] A. Tayebi and M. B. Zaremba, "Robust iterative learning control design is straightforward for uncertain LTI systems satisfying the robust performance condition," IEEE Transactions on Automatic Control, vol. 48, no. 1, pp. 101-106, 2003.

[14] R. Chi, D. Wang, Z. Hou, and S. Jin, "Data-driven optimal terminal iterative learning control," Journal of Process Control, vol. 22, no. 10, pp. 2026-2037, 2012.

[15] P. Janssens, G. Pipeleers, and J. Swevers, "A data-driven constrained norm-optimal iterative learning control framework for LTI systems," IEEE Transactions on Control Systems Technology, vol. 21, no. 2, pp. 546-551, 2013.

[16] Q. Yu and Z. Hou, "Data-driven predictive iterative learning control for a class of multiple-input and multiple-output nonlinear systems," Transactions of the Institute of Measurement and Control, vol. 38, no. 3, pp. 266-281, 2016.

[17] R. A. Gupta and M.-Y. Chow, "Networked control system: overview and research trends," IEEE Transactions on Industrial Electronics, vol. 57, no. 7, pp. 2527-2535, 2010.

[18] M. Moayedi, Y. K. Foo, and Y. C. Soh, "Adaptive Kalman filtering in networked systems with random sensor delays, multiple packet dropouts and missing measurements," IEEE Transactions on Signal Processing, vol. 58, no. 3, pp. 1577-1588, 2010. 
[19] H.-S. Ahn, K. L. Moore, and Y. Chen, "Stability of discretetime iterative learning control with random data dropouts and delayed controlled signals in networked control systems," in Proceedings of the 10th International Conference on Control, Automation, Robotics and Vision (ICARCV '08), pp. 757-762, December 2008.

[20] Y.-J. Pan, H. J. Marquez, T. W. Chen, and L. Sheng, "Effects of network communications on a class of learning controlled nonlinear systems," International Journal of Systems Science, vol. 40, no. 7, pp. 757-767, 2009.

[21] X. Bu, F. Yu, Z. Hou, and F. Wang, "Iterative learning control for a class of nonlinear systems with random packet losses," Nonlinear Analysis: Real World Applications, vol. 14, no. 1, pp. 567-580, 2013.

[22] Y. Geng, H.-S. Ahn, and X. Ruan, "Robustness of discretetime iterative learning control for networked control systems with data dropouts," in Proceedings of the IEEE International Symposium on Intelligent Control (ISIC '15), pp. 906-911, Sydney, Australia, September 2015.

[23] X. Ruan and Z. Li, "Convergence characteristics of PD-type iterative learning control in discrete frequency domain," Journal of Process Control, vol. 24, no. 12, pp. 86-94, 2014. 


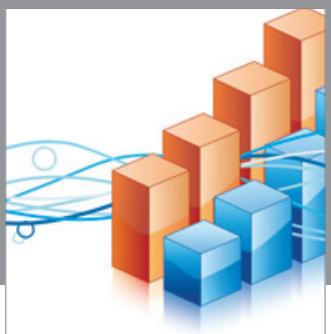

Advances in

Operations Research

vatem alat4

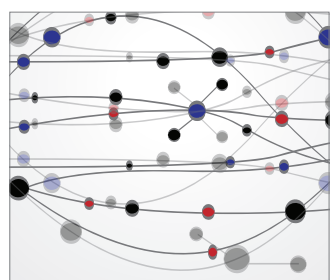

\section{The Scientific} World Journal
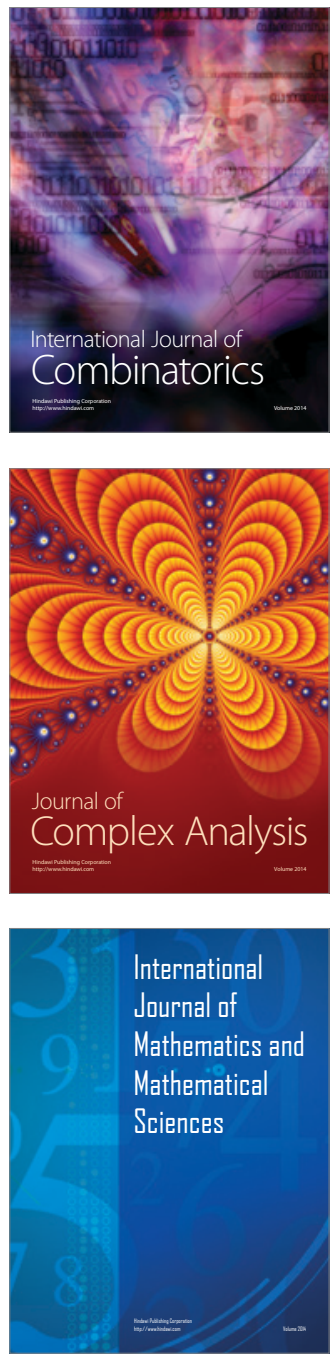
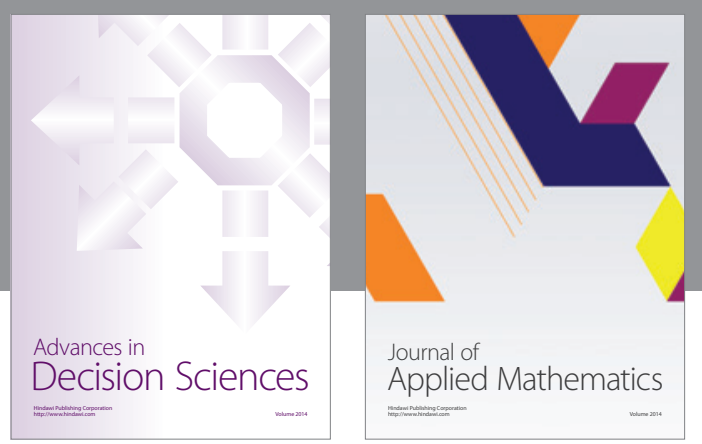

Algebra

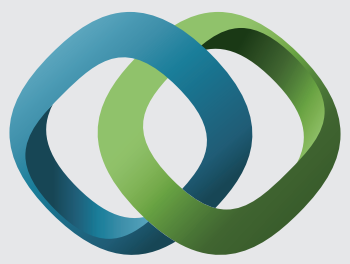

\section{Hindawi}

Submit your manuscripts at

https://www.hindawi.com
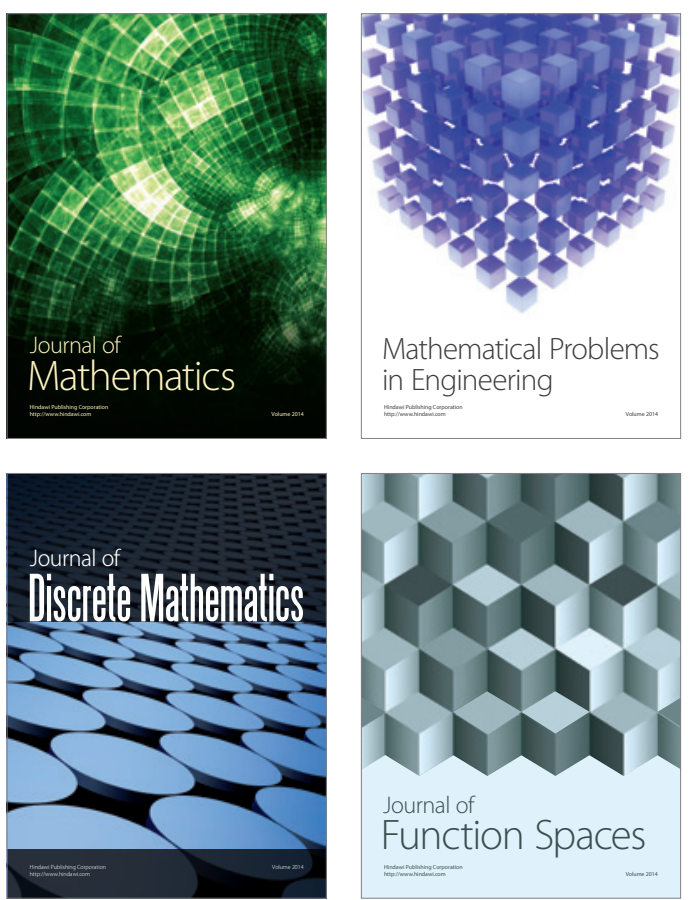

Mathematical Problems in Engineering
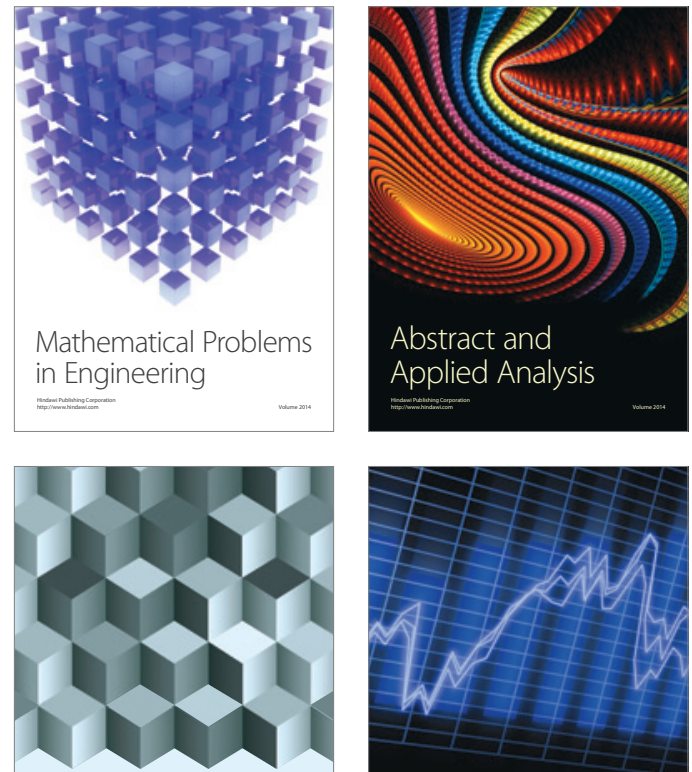

Journal of

Function Spaces

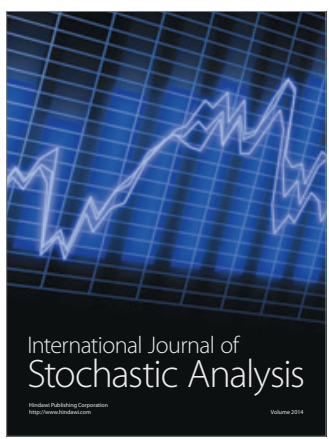

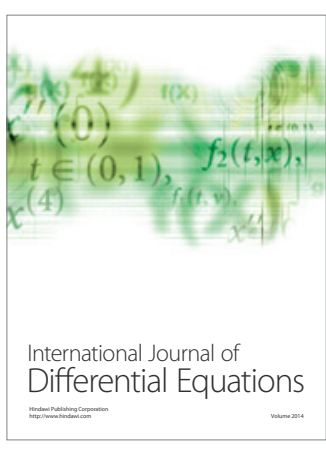
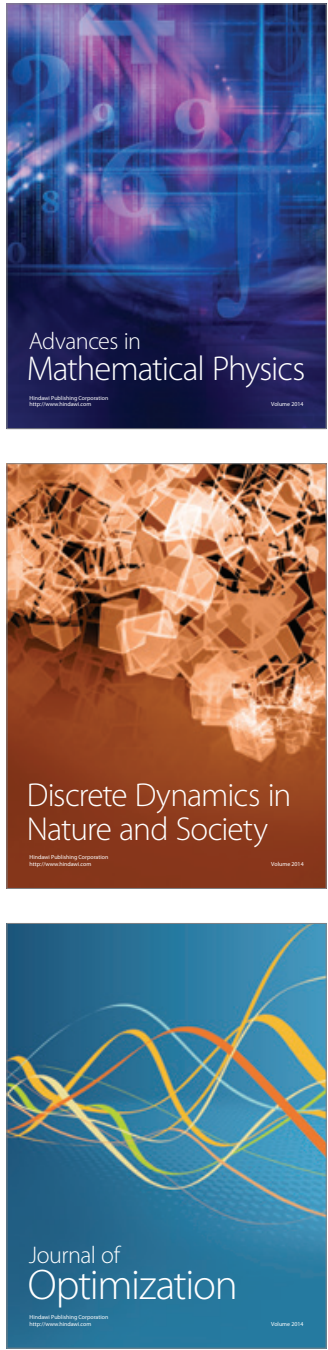